\title{
Suspension Performance Analysis and Experiment Optimization Based on K\&C Experiment
}

\author{
Li Fangli \\ Automotive Engineering institute, Jiangxi University of Technology, Nanchang 330098, China
}

Keywords: K\&C experiment, suspension performance, experiment analysis, automobile engineering Abstract. The research of automobile suspension is mainly used to improve handling stability of the whole automobile and driving performance, so this research subject is basically consisted by two parts: one is suspension performance field that mainly affects driving performance; the other one is the suspension performance field that mainly affects automobile handling stability. The paper analyzes and evaluates the fundamental principle, test methods of suspension performance experiment-K\&C experiment. Then test the sample automobile with automobile single axle $\mathrm{K} \& \mathrm{C}$ testbed to get test data and calculate by virtue of this test data and partial original data of MacPherson suspension model to get the basic data of suspension model sample automobile after adjusting.

\section{Introduction}

As one of the important assemblies of modern automobiles, the suspension connects automobile frame (or body) with the axle (or wheel) elastically. Its main function is to pass all forces and moments between wheels and the body, and to ease the impact load that road passes to the frame (or body), decreasing the caused vibration of carrier system to ensure driving comfort and the ideal moving performance of wheels with road load changing, as well as handling stability, to make the automobile get high speed capability.

In order to make the automobile have better driving comfort and handling stability, automobile suspension system design has to complete two major issues: 1. The design of suspension system, including suspension structure form design, suspension components transfer characteristics determination and other issues; 2 . Evaluation issue of suspension system performance, namely which basic parameters should be applied to systematically assess the merits of suspension system performance, how to evaluate the reasonableness of the change characteristics of suspension basic parameters, and which methods will be used to evaluate the change characteristic of the suspension system parameter index.

The study of automobile suspension system is mainly to improve automobile handling stability and driving comfort, so automobile suspension system research basically consists of two parts: one is suspension performance field that mainly affects driving performance; the other one is the suspension performance field that mainly affects automobile handling stability. The suspension system structure consists of elastic elements, damping elements, guides, buffer block and horizontal stabilizer. The first field focus on elastic element and damping element characteristics, that is, to study by taking road, tires, unsprung mass, suspension, sprung mass as a whole. Because it mainly researches the mechanical properties of the elastic element and damping element that affecting automobile performance under road force incentives, so we call it dynamics research of suspension 
system. Dynamics characteristics of suspension system directly affect the dynamics of a vehicle, so we can say that research of suspension dynamics is to research vehicle dynamics. The latter field is mainly on the suspension kinematics, that is, to study how the suspension guiding mechanism guide and restrain the movement of wheels, conduct wheel alignment and affect the kinematics characteristics of steering motion suspension parameters when the wheel and the vehicle body appear relative motion. The field research is called suspension kinematics research. In consideration of the impact on the suspension performance by other flexible connections like elastomeric bushing, the suspension kinematics is the suspension elastic kinematics. Suspension elastic kinematics is to describe the variation characteristics of main suspension parameters of wheels alignment caused by the force and moment between road surface and the tire. So, the suspension system kinematic should include suspension kinematics and elastic kinematics.

\section{Multi-body dynamics method}

In the 1970s, in order to analyze the kinematics and dynamics of the complex system composed of many objects, Robertson et al put forward different solutions successively, and the mathematical models they derived are applicable on computer modeling and calculation. This mechanics branch combined with classical rigid body dynamics, analytical mechanics and computer technology is multi-rigid-body system dynamics. It abstracts system inner pieces rigid body, to study their dynamics in a large range of spatial movement. Multi-body-system dynamics includes Newton Euler, Lagrange equation method, graph theory, Kane method, variational methods and spinor method.

In 80 s, based on multi-rigid-body dynamics, multi-flexible-body system dynamics developed rapidly and formed a systematic theory and method. Multi-flexible-body system dynamics takes the deformation of components and the interaction between flexible-body deformation and rigid motion of the whole system into account. The basic theories and methods of deriving multi- flexible-body system dynamics equations can be divided into three categories: The first category is the Newton Euler (Newton-Euler) vector mechanics method, the second is the analysis method of mechanics represented by Lagrangian (Lagrange) equation, and the third is based on the method of extreme value principle with minimal value nature, like Gaussian (Gauss) principle.

Multi-rigid-body system dynamics and multi-flexible -body system dynamics form multi-body system dynamics together. With the gradual development and improvement of multi-body system dynamics, a variety of methods and analysis software have also come out, including MSC.ADAMS, DADS and other famous software. In this paper, MSC.ADAMS software is used for dynamic analysis.

\section{Evaluation index for suspension $K \& C$ characteristics}

Camber angle. Camber angle formed by the crossing line of vehicle transverse plane of the wheel center and the wheel plane and the vehicle line of ground, i.e., to see the angle formed by the center line of the tire and the vertical line in front of the car, and it is positive outward, inward negative, as shown in Fig .1:

Camber angle can not only adapt to arch surface of the road, and different angle can change the tire contact with the ground and affect the wear condition of the tires, but its presence can also offset the amount of deformation of the suspension system after loading, thereby reducing uneven wear of 
the tire. In addition, as mentioned earlier, the hub outer end small bearing life can also be increased, reducing the load on the hub fastening nut.

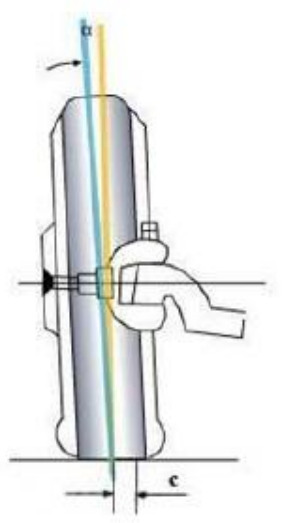

Figure 1. Camber angle

Toe angle. Because of the constraint of steering tie rod and axle, wheels cannot go outward, but edges inward phenomenon will appear on the ground, thus increasing tire wear. In order to avoid the adverse consequences due to the tapered rolling effect, make the two front wheels inward deflect appropriately to form toe angle of the front wheel. The distance from front edge of both sides wheel to $\mathrm{B}$ is less than the distance from the rear edge $\mathrm{A}$, and the difference between the distance from rear edge to $\mathrm{A}$ and the front edge to $\mathrm{B}$ is the Toe angle $\Delta$. When the front edge of the car is less than the rear edge, the toe is positive, otherwise it is negative. Horizontal diameter of the wheel and the vehicle's longitudinal symmetry plane form the toe angle. Fig .2:

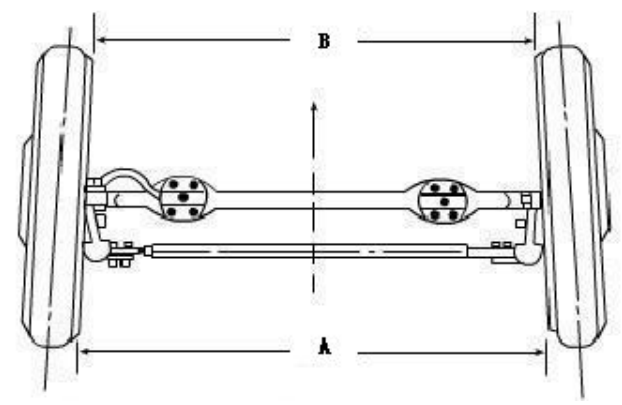

Figure 2. Toe angle

Caster Angle and Caster Moment Arm. Caster Angle is the included angle formed by the master pin axis and vertical line of ground within the longitudinal plane inside the car. The inclined angle toward to the back of vertical line is called positive caster; the inclined angle toward to the front is called negative caster. Usually, when a car is running, the caster angle value is positive.

\section{Longitudinal estimation of stability}

When the car is in braking and acceleration conditions, the inertial force will cause axle load transfer, accompanied by front and rear suspension deformation, appearing the phenomenon "dive and tail lifting" in braking and "front lifting and tail squat" in accelerating. The main parameters to evaluate these phenomena usually include: Dive Braking degree of the front suspension during braking, Lift Braking of rear suspension during braking, Lift Acceleration of front suspension during acceleration, and Squat Acceleration degree of rear suspension during acceleration. These parameter values reflect 
the constraint capacity of the suspension for wheel center vertical displacement during braking and acceleration directly, and reflect the constraint capacity of the suspension of car body pitching motion during braking and acceleration. In order to ensure the car has good resistance performance of pitching, the parameters abovementioned are expected to have less change in the process of acceleration and braking. However, the process of braking (or accelerating) operating conditions is accompanied by transfer of axle load, and car body pitching movement (or stretching and compression movement of suspension) is the result of braking force (or driving force) and axle load transfer interaction. For example, in the braking process, in terms of the front suspension, front axle load transfer (increasing) will make the wheel center perform close movement to car body, namely to resist car body produce diving effect. So it's not completely right to consider the four parameters of braking force (or driving force) for suspension against vehicle pitching, the axle load transfer of the vehicle's anti-pitching effect should also be considered. Thus, using following parameters to evaluate anti-dive ability is more accurate, namely front suspension Anti-Dive Braking, rear suspension Anti-Lift Braking, front suspension Anti-Lift Acceleration, and rear suspension Anti-Squat Acceleration. These four parameters reflect the proportional relation between the ability of suspension to resist body pitching movement under the effect of braking or diving force and the ability of axle load transfer making the body to produce pitching movement, better reflecting the suspension inhibiting car body pitching.

\section{Experiment method of suspension K\&C simulated analysis}

Parallel Wheel Travel Experiment. Parallel Wheel Travel is one of the basic methods of suspension kinematics simulation analysis. In the suspension motion simulation analysis, it simulates wheel vertical jump movement when wheels encounter an obstacle or uneven pavement. The left and right wheels will be applied the same travel, that is entering vertical jump amount with same size and same direction respectively, so that to achieve the change curve of wheel travel and the suspension parameters.

Opposite Wheel Travel Experiment. Opposite Wheel Travel is also one of the basic methods of suspension kinematics simulation analysis, similar with Parallel Wheel Travel Experiment. When taking suspension motion simulation analysis, namely simulating wheels encountering obstacles or uneven road surface casing vertical jump movement. The left and right wheels will be applied the same travel, that is entering vertical jump amount with same size and opposite direction respectively, so that to achieve the change curve of wheel travel and the suspension parameters.

Single Wheel Travel Experiment. Single Wheel Travel is different with Wheel Travel Experiment of the same direction, as the name suggests that the wheel of one side is fixed during simulation, applying vertical jump on the other side. For the simulation experiment of single suspension, the left and right wheels are independent without influencing each other, thus it's the same (anti) with Wheel Travel Experiment of the same direction; for the simulation of both side wheels, which will affect each other in jumping, so Single Wheel Travel Experiment is also needed except the abovementioned two experiments, to research the effect of one side wheel jumping on the other side suspension kinematics characteristics. The relation curve of wheel travel and suspension parameters is achieved through simulation.

Static Load Experiment. Static Load is a suspension elastic kinematics simulation to simulate the wheels' contact point with ground or wheel center suffering braking force, driving force, lateral force, aligning torque, and roll resisting moment and so on. In simulation analysis, according to the 
suspension characteristics difference to be analyzed, we can apply different force or torque to measure the changing relation curve of related elastic kinematics parameters with force or torque. It is mainly used to analyze the influence of the rubber bushings and other elastic connections on suspension performance, including the influence of various forces or moments on the important parameters like toe angle and camber angle.

Steering Experiment. Steering is a simulation experiment to simulate the effect of the vehicle steering process on suspension performance. It will simulate steering input by inputting starting steering angle and the maximum steering angle, to simulate the influence of steering process on suspension performance, to obtain the relative performance parameters curve.

\section{Acknowledgements}

This work was financially supported by the key subject building project (vehicle engineering) of Jiangxi University of Technology.

\section{References:}

[1] Chen J, Qin M, Jiang Y, et al. Modeling, Analysis and Optimization of the Twist Beam Suspension System[J]. SAE International Journal of Commercial Vehicles, 2015, 8(2015-01-0623).

[2] Li H, Jing X, Karimi H. Output-feedback based H-infinity control for active suspension systems with control delay[J]. IEEE Transactions on Industrial Electronics, 2014 (61): 436-446.

[3] Gu Z, Fei S, Zhao Y, et al. Robust control of automotive active seat-suspension system subject to actuator saturation[J]. Journal of Dynamic Systems, Measurement, and Control, 2014, 136(4): 041022 .

[4] Hatzell K B, Iwama E, Ferris A, et al. Capacitive deionization concept based on suspension electrodes without ion exchange membranes[J]. Electrochemistry Communications, 2014, 43: 18-21.

[5] Tian G, Zhang Y, Liu J H, et al. Double Wishbone Independent Suspension Parameter Optimization and Simulation[C]//Applied Mechanics and Materials. 2014, 574: 109-113.

[6] JIANG T, CHEN L, ZHANG X. Suspension Performance Analysis Based on Combination of Inerter-Spring-Damper[J]. Journal of Chongqing University of Technology (Natural Science), 2014, 12: 007.

[7] Kamarei F, Movaghari F, Ghaffari A, et al. Development of a stability-indicating high performance liquid chromatography method for assay of erythromycin ethylsuccinate in powder for oral suspension dosage form[J]. Arabian Journal of Chemistry, 2014, 7(6): 1079-1085.

[8] Gadhvi B, Savsani V. Passive Suspension Optimization Using Teaching Learning Based Optimization and Genetic Algorithm Considering Variable Speed Over a Bump[C]//ASME 2014 International Mechanical Engineering Congress and Exposition. American Society of Mechanical Engineers, 2014: V04AT04A018-V04AT04A018.

[9] Najlah M, Parveen I, Alhnan M A, et al. The effects of suspension particle size on the performance of air-jet, ultrasonic and vibrating-mesh nebulisers[J]. International journal of pharmaceutics, 2014, 461(1): 234-241. 
[10]Wu J L. A Simultaneous Mixed LQR/Hळ Control Approach to the Design of Reliable Active Suspension Controllers[J]. Asian Journal of Control, 2014. 